\title{
In Memory of Aleksey Petrovich Popryadukhin (February 28, 1935-November 4, 2007)
}

PACS numbers: 01.60.+q

DOI: $10.1134 / \mathrm{S} 1063780 \mathrm{X} 08060111$

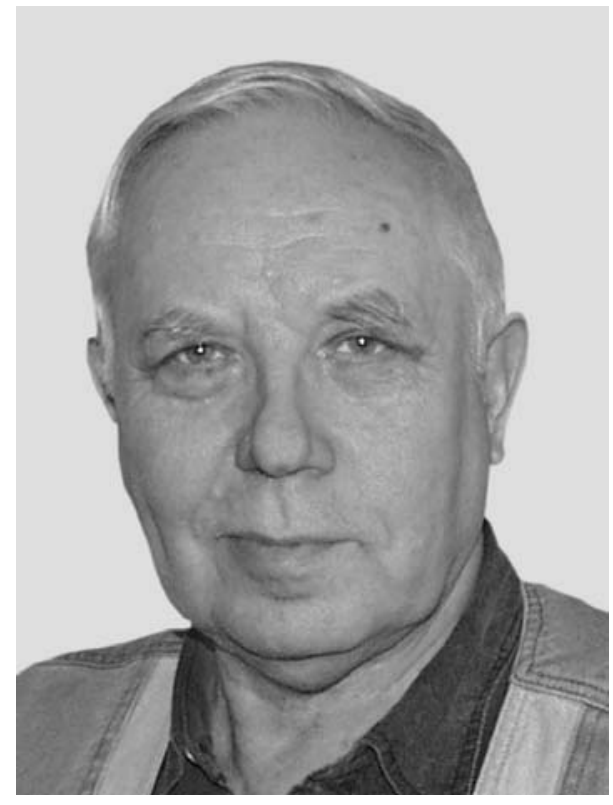

Aleksey Petrovich Popryadukhin, a doctor of physical and mathematical sciences and a principal researcher of the Department of the Physics of Tokamak Reactors at the Troitsk Institute for Innovation and Fusion Research, passed away on November 4, 2007, after an extended illness.

We have lost a man of exceptional ability and generous soul; a kind-hearted and sympathetic friend; and an eminent physicist, well recognized both in Russia and abroad.

After graduating from the Moscow Engineering Physics Institute in 1958, Popryadukhin began to work at the Lebedev Physical Institute of the Russian Academy of Sciences. There, he exhibited the rare talent of combining theoretical and experimental research. The results obtained by him went down in the history of plasma physics. In the 1960s, he carried out a series of studies on the properties of magnetic surfaces in toroidal systems and the trajectories of charged particles in the presence of electric fields. These studies laid the basis for his candidate's thesis. He was one of the authors of a well-known experimental work on measur- ing the structure of magnetic surfaces in the L-1 stellarator by using an original multipass electron technique. In those studies, the existence of stellarator magnetic surfaces was proved for the first time, their structure at different values of the rotational transform was investigated, and the occurrence of magnetic islands near rational magnetic surfaces was revealed experimentally. Later, together with S.N. Popov, he proposed to construct modular stellarator configurations by using elliptic magnetic coils. This idea was experimentally verified in the Torus-2 modular stellarator (a prototype of a new class of closed magnetic confinement systems), which was created at the Lebedev Institute, and laid down in the basis of one of the largest contemporary stellarators-the $\mathrm{W} 7-\mathrm{X}$ stellarator, which is now under construction in Germany.

In 1974, he moved to the Kurchatov Institute, where he proposed an idea of creating a tokamak with an original toroidal divertor. This idea was implemented under his leadership in the T0-2 tokamak - a unique device of this type.

In 1986, Popryadukhin moved to the Troitsk Institute for Innovation and Fusion Research (a branch of the Kurchatov Institute), where he headed a group dealing with magnetic measurements. Along with these studies, he analyzed theoretically how quasi-steady helical magnetic perturbations influence tokamak discharges and, in 1995, defended a doctoral thesis on this challenging problem in tokamak physics.

An important problem with which he was occupied throughout his scientific life was the influence of the local electric field perturbations on plasma transport in closed confinement systems. He initiated relevant experiments, which, however, were interrupted by his heavy illness.

We have lost a true friend; an eminent and faithful enthusiast of science; a talented teacher; and a courageous man, who fought illness to the end. His life is a brilliant example of firmy following one's principles. With deep gratitude, we will always keep his memory in our hearts. 